INPLASY

PROTOCOL

To cite: Wang et al. Efficacy and safety of warm needle treatment for scapulohumeral periarthritis: A protocol for systematic review and metaanalysis. Inplasy protocol 2020100049. doi:

10.37766/inplasy2020.10.0049

Received: 14 October 2020

Published: 14 October 2020

Corresponding author: Sun Qing

13820290606@163.com

Author Affiliation:

First Teaching Hospital of

Tianjin University of Traditional

Chinese Medicine

Support: National Natural

Science Found.

Review Stage at time of this submission: The review has not yet started.

Conflicts of interest:

The authors have no conflicts of interest to disclose.

\section{Efficacy and safety of warm needle treatment for scapulohumeral periarthritis: A protocol for systematic review and meta-analysis}

Wang, X1; Hai, X²; Jiang, D; Yin, L4; Li, H5; Wang, Q6; Liu, F7; $\mathrm{Xu}, \mathrm{G} 8$; Sun, $\mathrm{Q}^{9}$.

Review question / Objective: o evaluate the effectiveness and safety of warm needle acupuncture (WNA) treatment for Scapulohumeral periarthritis (SP).

Condition being studied: Scapulohumeral periarthritis is a common and disabling musculoskeletal disease in middleaged people. It usually refers to shoulder pain syndrome caused by soft tissue damage around the shoulder joint and restricted motor function, which seriously affects the life and work of patients. Bring a lot of inconvenience and pain. In recent years, many literatures reported that WNA has a definite effect on SP. Relevant randomized controlled trials (RCTs) will be searched from the databases of Pubmed, the Cochrane Library, Embase, CNKI, Wanfang Database, CBM and VIP Database from their inception to September 2021. Two reviewers will independently select studies, collect data, and assess the methodology quality by the Cochrane risk of bias tool. The Stata $\mathbf{1 4 . 0}$ will be used for meta-analysis. This study will provide an assessment of the current state of WNA for the SP, aiming to show the efficacy and safety of WNA treatment.

INPLASY registration number: This protocol was registered with the International Platform of Registered Systematic Review and Meta-Analysis Protocols (INPLASY) on 14 October 2020 and was last updated on 14 October 2020 (registration number INPLASY2020100049).

\section{INTRODUCTION}

Review question / Objective: To evaluate the effectiveness and safety of warm needle acupuncture (WNA) treatment for Scapulohumeral periarthritis (SP).
Condition being studied: Scapulohumeral periarthritis is a common and disabling musculoskeletal disease in middle-aged people. It usually refers to shoulder pain syndrome caused by soft tissue damage 
around the shoulder joint and restricted motor function, which seriously affects the life and work of patients. Bring a lot of inconvenience and pain. In recent years, many literatures reported that WNA has a definite effect on SP. Relevant randomized controlled trials (RCTs) will be searched from the databases of Pubmed, the Cochrane Library, Embase, CNKI, Wanfang Database, CBM and VIP Database from their inception to September 2021. Two reviewers will independently select studies, collect data, and assess the methodology quality by the Cochrane risk of bias tool. The Stata 14.0 will be used for metaanalysis. This study will provide an assessment of the current state of WNA for the SP, aiming to show the efficacy and safety of WNA treatment.

\section{METHODS}

Search strategy: We will search for Pubmed, the Cochrane Library, EMbase, CNKI, Wanfang Database, CBM and VIP Database from its inception to September 2021 with a language restriction on Chinese or English.

Participant or population: Patients diagnosed with SP are not restricted by age, sex, race, occupation, education, aetiology, and severity etc. The diagnostic criteria must be clear.

Intervention: WNA, or WNA combine with other conventional treatments.

Comparator: The control group was given conventional treatments such as drugs, placebo, sham acupuncture and no treatment, etc.

Study designs to be included: All randomized controlled trials (RCT) evaluate the effectiveness and safety of WNA in the treatment of SP will be included.

Eligibility criteria: 1.Type of studies. All randomized controlled trials (RCT) evaluate the effectiveness and safety of WNA in the treatment of SP will be included. 2.Types of participants. Patients diagnosed with SP are not restricted by age, sex, race, occupation, education, aetiology, and severity etc. The diagnostic criteria must be clear. 3.Types of intervention. WNA, or WNA combine with other conventional treatments were used as the intervention measures in the treatment group, while the control group was given conventional treatments such as drugs, placebo, sham acupuncture and no treatment, etc. 4.Types of outcome measures. 4.1. Primary outcomes. The primary efficacy outcomes measure will be as follows: effective rate, visual analogue scale (VAS) score. 4.2. Secondary outcomes. The secondary outcomes measure will include the Constant-Murley score, Japanese Orthopaedic Association scores, adverse events.

Information sources: We will search for Pubmed, the Cochrane Library, Embase, CNKI, Wanfang Database, CBM and VIP Database from its inception to September 2021 with a language restriction on Chinese or English.

Main outcome(s): Effective rate, visual analogue scale (VAS) score.

Additional outcome(s): Constant-Murley score, Japanese Orthopaedic Association scores, adverse events.

Data management: The literature was screened independently by two evaluators based on pre-defined inclusion and exclusion criteria and then cross-over, with discussion to resolve any disagreements or consultation with a third evaluator to make a decision if they arose. The following information and data were extracted from each included clinical trial: authors, time of publication, randomization method, blinding, number of cases of observation, diagnostic criteria, interventions and controls, duration of treatment, efficacy indicators, and follow-up.

Quality assessment / Risk of bias analysis: The quality of the included literature was assessed by two evaluators using the risk of bias assessment tool recommended in the Cochrane Handbook 5.3, and if disagreements arose they were resolved by 
discussion or a third evaluator was consulted for a decision. Criteria included: correct use of randomisation; correct use of allocation concealment; correct use of blinding of patients; correct use of blinding of researchers; completeness of results and data; selective reporting of results; and presence of relevant bias.

Strategy of data synthesis: Meta-analysis was performed using Rev Man $\mathbf{5 . 3}$ software. Relative risk (RR) and 95\% confidence interval were used for the count data, SMD and $95 \%$ confidence interval were used for the measurement data as effect measures; if there was heterogeneity among the intervention protocols included in the studies, the heterogeneity test was performed between the studies using the karyotype test (test for $a=0.05$ ), when $P<$ 0.1 or $12>50 \%$. perceived heterogeneity, using random-effects should model to calculate the OR of the overall result; conversely, the fixed-effects model was used to calculate, and publication bias was analyzed using funnel plots. Subgroup analyses were performed based on factors that could be heterogeneous, and sensitivity analyses were performed when heterogeneity originated from low-quality studies.

Subgroup analysis: If the included studies are highly heterogeneous, we will perform a subgroup analysis based on age, sample size, methodological quality, etc.

Sensibility analysis: If heterogeneity is significant, we will conduct a sensitivity analysis to assess the robustness and quality of the findings by excluding each included study individually and varying the study's impact scale.

Language: Chinese and English.

Country(ies) involved: China.

Keywords: warm needle acupuncture, Scapulohumeral periarthritis, metaanalysis, systematic review, protocol.

Contributions of each author:

Author 1 - Xiaoyu Wang.
Author 2 - Xinghua Hai.

Author 3 - Dongli Jiang.

Author 4 - Lianjun Yin.

Author 5 - Huanan Li.

Author 6 - Qi Wang.

Author 7 - Fang Liu.

Author 8 - Guoqiang Xu.

Author 9 - Qing Sun. 